\title{
On the one-dimensional parabolic obstacle problem with variable coefficients
}

\author{
A. Blanchet ${ }^{* \dagger}$, J. Dolbeault,$*$ R. Monneau ${ }^{\dagger}$
}

\begin{abstract}
This note is devoted to continuity results of the time derivative of the solution to the onedimensional parabolic obstacle problem with variable coefficients. It applies to the smooth fit principle in numerical analysis and in financial mathematics. It relies on various tools for the study of free boundary problems: blow-up method, monotonicity formulae, Liouville's results.
\end{abstract}

AMS Classification: 35R35.

Keywords: parabolic obstacle problem, free boundary, blow-up, Liouville's result, monotonicity formula, smooth fit.

\section{Introduction}

Consider a parabolic obstacle problem in an open set. We look for local properties, which do not depend on the boundary conditions and the initial conditions, but only depend on the equation in the interior of the domain. Consider a function $u$ with a one-dimensional space variable $x$ in $Q_{1}(0)$ where by $Q_{r}\left(P_{0}\right)$ we denote the parabolic box of radius $r$ and of centre $P_{0}=\left(x_{0}, t_{0}\right)$ :

$$
Q_{r}\left(P_{0}\right)=\left\{(x, t) \in \mathbb{R}^{2}, \quad\left|x-x_{0}\right|<r, \quad\left|t-t_{0}\right|<r^{2}\right\} .
$$

Assume that $u$ is a solution of the one-dimensional parabolic obstacle problem with variable coefficients:

$$
\left\{\begin{array}{l}
a(x, t) u_{x x}+b(x, t) u_{x}+c(x, t) u-u_{t}=f(x, t) \cdot \mathbb{1}_{\{u>0\}} \quad \text { a.e. in } Q_{1}(0) \\
u \geq 0 \quad \text { a.e. in } Q_{1}(0)
\end{array}\right.
$$

${ }^{*}$ CEREMADE, Université Paris Dauphine, place de Lattre de Tassigny, 75775 Paris Cédex 16, France

${ }^{\dagger}$ CERMICS, Ecole Nationale des Ponts et Chaussées, 6 et 8 avenue Blaise Pascal, Cité Descartes Champs-sur-Marne, 77455 Marne-la-Vallée Cédex 2, France 
where $u_{t}, u_{x}, u_{x x}$ respectively stand for $\frac{\partial u}{\partial t}, \frac{\partial u}{\partial x}, \frac{\partial^{2} u}{\partial x^{2}}$, and $\mathbb{1}_{\{u>0\}}$ is the characteristic function of the positive set of $u$. Here the free boundary $\Gamma$ is defined by

$$
\Gamma=(\partial\{u=0\}) \cap Q_{1}(0) .
$$

To simplify the presentation, we assume that the coefficients

$$
a, b, c \text { and } f \text { are } C^{1} \text { in }(x, t) \text {, }
$$

but Hölder continuous would be sufficient in what follows.

A natural assumption is that the differential operator is uniformly elliptic, i.e. the coefficient $a$ is bounded from below by zero. If we do not make further assumptions on $a$ and on $f$, we cannot expect any good property of the free boundary $\Gamma$. Suppose that:

$$
\exists \delta>0, \quad a(x, t) \geq \delta, f(x, t) \geq \delta \text { a.e. in } Q_{1}(0)
$$

Up to a reduction of the size of the box (see [4]), any weak solution $u$ of (1) has a bounded first derivative in time and bounded first and second derivatives in space. Assume therefore that this property holds on the initial box:

$$
|u(x, t)|,\left|u_{t}(x, t)\right|,\left|u_{x}(x, t)\right| \text { and }\left|u_{x x}(x, t)\right| \text { are bounded in } Q_{1}(0) \text {. }
$$

This problem is a generalisation to the case of an operator with variable coefficients of Stefan's problem (case where the parabolic operator is $\partial^{2} / \partial x^{2}-\partial / \partial t$ ). Stefan's problem describes the interface of ice and water (see $[10,13,8]$ ). The problem with variable coefficients arises in the pricing of american options in financial mathematics (see $[3,2,14,11,9,15,1])$.

If $P$ is a point such that $u(P)>0$, by standard parabolic estimates $u_{t}$ is continuous in a neighbourhood of $P$. On the other hand if $P$ is in the interior of the region $\{u=0\}$, $u_{t}$ is obviously continuous. The only difficulty is therefore the regularity on the free boundary $\Gamma$. By assumption $u_{t}$ is bounded but may be discontinuous on $\Gamma$. The regularity of $u_{t}$ is a crucial question to apply the "smooth-fit principle" which amounts to the $C^{1}$ continuity of the solution at the free boundary. This principle is often assumed, especially in the papers dealing with numerical analysis (see P. Dupuis and H. Wang [6] for example).

In a recent work L. Caffarelli, A. Petrosyan and H. Shagholian [5] prove the $\mathcal{C}^{\infty}$ regularity of the free boundary locally around some points which are energetically characterised, without any sign assumption neither on $u$ nor on its time derivative. This result holds in higher dimension but in the case of constant coefficients. We use tools similar to the ones of [5] and the ones the last author developed previously for the elliptic obstacle problem in [12]. Our main result is the following: 
Theorem 1.1 (Continuity of $u_{t}$ for almost every time) Under assumptions (1)-(2)(3)-(4), for almost every time $t$, the function $u_{t}$ is continuous on $Q_{1}(0)$.

This result is new, even in the case of constant coefficients. The continuity of $u_{t}$ cannot be obtained everywhere in $t$, as shown by the following example. Let $u(x, t)=\max \{0,-t\}$. It satisfies $u_{x x}-u_{t}=\mathbb{1}_{\{u>0\}}$ and its time derivative is obviously discontinuous at $t=0$.

If additionally we assume that $u_{t} \geq 0$ we achieve a more precise result:

Theorem 1.2 (Continuity of $u_{t}$ for all $t$ when $u_{t} \geq 0$ ) Under assumptions (1)-(2)(3)-(4), if $u_{t} \geq 0$ in $Q_{1}(0)$ then $u_{t}$ is continuous everywhere in $Q_{1}(0)$.

The assumption that $u_{t} \geq 0$ can be established in some special cases (special initial conditions, boundary conditions, and time independent coefficients). See for example the results of Friedman [7], for further results on the one-dimensional parabolic obstacle problem with particular initial conditions.

In Section 2 we introduce blow-up sequences, which are a kind of zoom at a point of the free boundary. They converge, up to a sub-sequence, to a solution on the whole space of the obstacle problem with constant coefficients. Thanks to a monotonicity formula for an energy we prove in Section 3 that the blow-up limit is scale-invariant. This allows us to classify in Section 4 all possible blow-up limits in a Liouville's theorem. Then we sketch the proof of Theorem 1.1. We even classify energetically the points of the free boundary into the set of regular and singular points. In Section 5 we prove the uniqueness of the blow-up limit at singular points. Then we give the sketch of the proof of the Theorem 1.2. For further details we refer to [4].

\section{The notion of blow-up}

Given a point $P_{0}=\left(x_{0}, t_{0}\right)$ on the free boundary $\Gamma$, we can define the blow-up sequence by

$$
u_{P_{0}}^{\varepsilon}(x, t)=\frac{u\left(x_{0}+\varepsilon x, t_{0}+\varepsilon^{2} t\right)}{\varepsilon^{2}}, \varepsilon>0 .
$$

Roughly speaking the action of this rescaling is to zoom on the free boundary at scale $\varepsilon$ (see figure 1).

By assumption, $u\left(P_{0}\right)=0$. Because $u$ is non-negative, we also have $u_{x}\left(P_{0}\right)=0$. Moreover $u_{P_{0}}^{\varepsilon}$ has a bounded first derivative in time and bounded second derivatives in space. For this reason, using Ascoli-Arzelà's theorem, we can find a sequence $\left(\varepsilon_{n}\right)_{n}$ which converges to zero such that $\left(u_{P_{0}}^{\varepsilon_{n}}\right)_{n}$ converges on every compact set of $\mathbb{R}^{2}=\mathbb{R}_{x} \times \mathbb{R}_{t}$ to a function $u^{0}$ (called the blow-up limit) and which a priori depends on the choice of the sequence $\left(\varepsilon_{n}\right)_{n}$. 


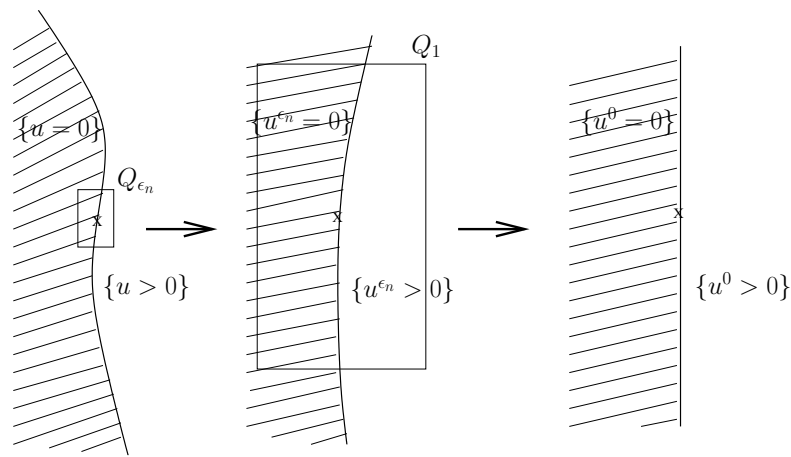

Figure 1: Blow-up

The limit function $u^{0}$ satisfies the parabolic obstacle problem with constant coefficients on the whole space-time:

$$
a\left(P_{0}\right) u_{x x}^{0}-u_{t}^{0}=f\left(P_{0}\right) \cdot \mathbb{1}_{\{u>0\}} \quad \text { in } \mathbb{R}^{2} .
$$

By the non-degeneracy assumption (3), it is possible to prove that $0 \in \partial\left\{u^{0}=0\right\}$.

To characterise the blow-up limit $u^{0}$, we need to come back to the original equation satisfied by $u$ and to obtain additional estimates. In order to simplify the presentation we make a much stronger assumption on $u$ : assume that $u$ is a solution on the whole space-time of the equation with constant coefficients $a \equiv 1, f \equiv 1, b \equiv 0$ and $c \equiv 0$ :

$$
u_{x x}-u_{t}=\mathbb{1}_{\{u>0\}} \text { in } \mathbb{R}^{2} .
$$

Without this assumption, all tools have to be localised. See [4] for more details.

\section{A monotonicity formula for energy}

For every time $t<0$, we define the quantity

$$
\mathcal{E}(t ; u)=\int_{\mathbb{R}}\left\{\frac{1}{-t}\left(\left|u_{x}(x, t)\right|^{2}+2 u(x, t)\right)-\frac{1}{t^{2}} u^{2}(x, t)\right\} G(x, t) d x
$$

where $G$ satisfies the backward heat equation $G_{x x}+G_{t}=0$ in $\{t<0\}$ and is given by

$$
G(x, t)=\frac{1}{2 \sqrt{\pi(-t)}} \exp \left(\frac{-x^{2}}{4(-t)}\right) .
$$

Theorem 3.1 (Monotonicity formula for energy) Assume that $u$ is a solution of (6). The function $\mathcal{E}$ is non-increasing in time for $t<0$, and satisfies

$$
\frac{d}{d t} \mathcal{E}(t ; u)=-\frac{1}{2(-t)^{3}} \int_{\mathbb{R}}|\mathcal{L} u(x, t)|^{2} G(x, t) d x
$$


where

$$
\mathcal{L} u(x, t)=-2 u(x, t)+x \cdot u_{x}(x, t)+2 t \cdot u_{t}(x, t) .
$$

A similar but different energy is introduced in $[5,16]$.

Corollary 3.2 (Homogeneity of the blow-up limit) Any blow-up limit $u^{0}$ of $\left(u_{P_{0}}^{\varepsilon_{n}}\right)_{n}$ defined in (5), satisfies

$$
u^{0}\left(\lambda x, \lambda^{2} t\right)=\lambda^{2} u^{0}(x, t) \quad \text { for every } \quad x \in \mathbb{R}, t<0, \lambda>0 .
$$

Proof. We prove it in the case $P_{0}=0$. The crucial property is the scale-invariance of $\mathcal{E}$ :

$$
\mathcal{E}\left(\varepsilon_{n}^{2} t ; u\right)=\mathcal{E}\left(t ; u_{0}^{\varepsilon_{n}}\right) .
$$

Taking the limit $\varepsilon_{n} \rightarrow 0$, we get

$$
\mathcal{E}\left(0^{-} ; u\right):=\lim _{\tau \rightarrow 0} \tau<0
$$

From the monotonicity formula (7), we get $\mathcal{L} u^{0}(x, t)=0$ for $t<0$. This implies the homogeneity (8) of $u^{0}$ for $t<0$.

\section{A Liouville's theorem and consequences}

Let

$$
\begin{gathered}
v_{+}(x, t)=\frac{1}{2}(\max \{0, x\})^{2}, \\
v_{-}(x, t)=\frac{1}{2}(\max \{0,-x\})^{2},
\end{gathered}
$$

and for $m \in[-1,0]$

$$
v_{m}(x, t)= \begin{cases}m t+\frac{1+m}{2} x^{2} & \text { if } \quad t \leq 0, \\ t V_{m}\left(\frac{|x|}{t}\right)>0 & \text { if } \quad 0<t<C_{m} \cdot x^{2}, \\ 0 & \text { if } \quad t \geq C_{m} \cdot x^{2},\end{cases}
$$

where the coefficient $C_{m}$ is an increasing function of $m$, satisfying $C_{m}=0$ if $m=-1$, and $C_{m}=+\infty$, if $m=0$. The precise expression of $V_{m}$ is given in [4]. In particular we get $v_{-1}(x, t)=\max \{0,-t\}$ and $v_{0}(x, t)=\frac{1}{2} x^{2}$.

Theorem 4.1 (Classification of global homogeneous solutions in $\mathbb{R}^{2}$ ) Let $u^{0} \not \equiv 0$ be a non-negative solution of (6) satisfying the homogeneity condition (8). Then $u^{0}$ is one of $v_{+}, v_{-}$or $v_{m}$ for some $m \in[-1,0]$. 


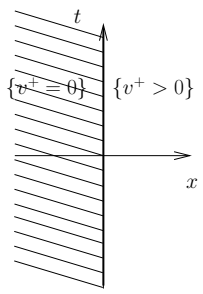

$v_{+}$

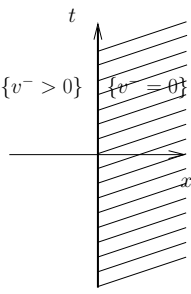

$v_{-}$

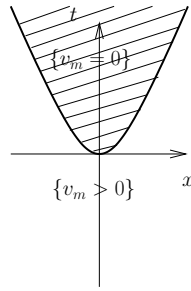

$v_{m}, m \in(-1,0)$
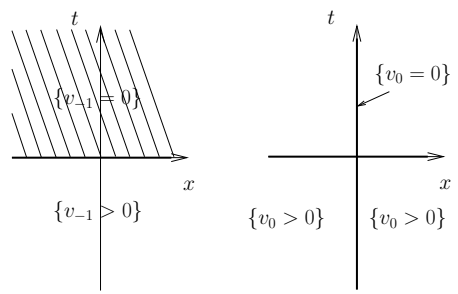

$v_{-1}$

$v_{0}$

Figure 2: Solutions of Theorem 4.1

Similar versions of this theorem are also proved in [5].

Theorem 1.2 is a consequence of Theorem 4.1. Every blow-up limit satisfies $u_{t}^{0} \leq 0$. A more detailed analysis leads to

$$
\liminf _{P \rightarrow P_{0}} u_{t}(P) \leq 0 .
$$

From the assumption $u_{t} \geq 0$ we so infer that $u_{t}=0$.

We also have an energy criterion to characterise points of the free boundary

Theorem 4.2 (Regular and singular points) Let $u$ be a solution of (6). Then either $\mathcal{E}\left(0^{-} ; u\right)=\sqrt{2}$ or $\mathcal{E}\left(0^{-} ; u\right)=\sqrt{2} / 2$.

In the first case $\left(\right.$ i.e. $\left.\mathcal{E}\left(0^{-} ; u\right)=\sqrt{2}\right) P_{0}$ is called a singular point. Otherwise (i.e. $\left.\mathcal{E}\left(0^{-} ; u\right)=\sqrt{2} / 2\right), P_{0}$ is a regular point.

Proof. By (9) we have $\mathcal{E}\left(0^{-} ; u\right)=\mathcal{E}\left(-1 ; u^{0}\right)$. Blow-up limits have been classified in Theorem 4.1. A simple calculation gives $\mathcal{E}\left(-1 ; v_{+}\right)=\mathcal{E}\left(-1 ; v_{+}\right)=\sqrt{2} / 2$, and $\mathcal{E}\left(-1 ; v_{m}\right)=\sqrt{2}$ for every $m \in[-1,0]$.

\section{A monotonicity formula for singular points}

One of the crucial idea of [12] can be adapted to the parabolic framework.

Theorem 5.1 (Monotonicity formula for singular points) Let $u$ be a solution of (6) and assume that $P_{0}=0$ is a singular point. For any $m \in[-1,0]$ the function

$$
t \mapsto \Phi^{v_{m}}(t ; u)=\int_{\mathbb{R}} \frac{1}{t^{2}}\left(u(x, t)-v_{m}(x, t)\right)^{2} G(x, t) d x, \quad t<0
$$

is non-increasing.

As a consequence $\lim _{\tau \rightarrow 0, \tau<0} \Phi^{v_{m}}(t ; u):=\Phi^{v_{m}}\left(0^{-} ; u\right)$ is well defined. 
Corollary 5.2 (Blow-up limit at singular points) Under the assumption of Theorem 5.1, there exists a real $m \in[-1,0]$ such that any blow-up limit of $u$ at 0 is equal to $v_{m}$.

Proof. Consider two sequences $\left(\varepsilon_{n}\right)_{n}$ and $\left(\tilde{\varepsilon}_{n}\right)_{n}$ converging to 0 such that $\left(u_{0}^{\varepsilon_{n}}\right)_{n}$ and $\left(u_{0}^{\tilde{\varepsilon}_{n}}\right)_{n}$ respectively converge to two blow-up limits $u^{0}=v_{m}$, for some $m \in[0,1]$, and $\tilde{u}^{0}$. Using the scale-invariance we get

$$
\Phi^{v_{m}}\left(-1 ; u_{0}^{\varepsilon_{n}}\right)=\Phi^{v_{m}}\left(-\varepsilon_{n}^{2} t ; u\right) \quad \text { and } \quad \Phi^{v_{m}}\left(-1 ; u_{0}^{\tilde{\varepsilon}_{n}}\right)=\Phi^{v_{m}}\left(-\tilde{\varepsilon}_{n}^{2} t ; u\right) .
$$

Passing to the limit in the scale-invariance we obtain

$$
0=\Phi^{v_{m}}\left(-1 ; u^{0}\right)=\Phi^{v_{m}}\left(0^{-} ; u\right)=\Phi^{v_{m}}\left(-1 ; \tilde{u}^{0}\right) .
$$

This proves that $\tilde{u}^{0}=v_{m}=u^{0}$, i.e. the uniqueness of the blow-up limit.

Heuristically the singular points whose blow-up limit is $v_{m}$ with $m \in[-1,0)$ have a free boundary with horizontal tangent in the $(x, t)$-plane: this almost never occurs. On the other hand the singular points with $v_{0}$ as blow-up limit and the regular points have a blow-up limit which satisfies $u_{t}^{0}=0$. This last argument can be refined to show that the time derivative of $u$ is continuous at such points, and consequently for almost every time. This proves Theorem 1.1.

\section{Acknowledgements}

We thank D. Lamberton and B. Lapeyre for their comments and suggestions. This study has been partially supported by the ACI NIM "EDP et finance, \# 2003-83".

(C) 2004 by the authors. This paper may be reproduced, in its entirety, for non-commercial purposes.

\section{References}

[1] Y. AchDou, An inverse problem for parabolic variational inequalities in the calibration of american options. to appear in SIAM J. Control Optim.

[2] A. Bensoussan And J.-L. Lions, Applications des inéquations variationnelles en contrôle stochastique, Dunod, Paris, 1978. Méthodes Mathématiques de l'Informatique, No. 6.

[3] F. Black and M. Scholes, The pricing of options and corporate liabilies, J. Polit. Econ., 81 (1973), pp. 637-659. 
[4] A. Blanchet, J. Dolbeault, and R. Monneau, On the one-dimensional parabolic obstacle problem with variable coefficients. Application to the pricing of american options. In preparation.

[5] L. Caffarelli, A. Petrosyan, and H. Shahgholian, Regularity of a free boundary in parabolic potential theory. Preprint.

[6] P. Dupuis And H. Wang, Optimal stopping with random intervention times, Adv. in Appl. Probab., 34 (2002), pp. 141-157.

[7] A. Friedman, Parabolic variational inequalities in one space dimension and smoothness of the free boundary, J. Functional Analysis, 18 (1975), pp. 151-176.

[8] — - Variational principles and free-boundary problems, Pure and Applied Mathematics, John Wiley \& Sons Inc., New York, 1982. A Wiley-Interscience Publication.

[9] P. Jaillet, D. Lamberton, and B. Lapeyre, Variational inequalities and the pricing of American options, Acta Appl. Math., 21 (1990), pp. 263-289.

[10] D. Kinderlehrer and G. Stampacchia, An introduction to variational inequalities and their applications, vol. 88 of Pure and Applied Mathematics, Academic Press Inc. [Harcourt Brace Jovanovich Publishers], New York, 1980.

[11] D. Lamberton And B. Lapeyre, Introduction au calcul stochastique appliqué à la finance, Ellipses Édition Marketing, Paris, second ed., 1997.

[12] R. Monneau, On the number of singularities for the obstacle problem in two dimensions, J. Geom. Anal., 13 (2003), pp. 359-389.

[13] J.-F. Rodrigues, Obstacle problems in mathematical physics, vol. 134 of NorthHolland Mathematics Studies, North-Holland Publishing Co., Amsterdam, 1987. Notas de Matemática [Mathematical Notes], 114.

[14] P. Van Moerbeke, An optimal stopping problem with linear reward, Acta Math., 132 (1974), pp. 111-151.

[15] S. Villeneuve, Options américaines dans un modèle de Black-Scholes multidimensionnel, PhD thesis, Université De Marne la Vallée, 1999.

[16] G. S. WeIss, Self-similar blow-up and Hausdorff dimension estimates for a class of parabolic free boundary problems, SIAM J. Math. Anal., 30 (1999), pp. 623-644 (electronic). 